\title{
Hybrid Quantization: From Bianchi I to the Gowdy Model
}

\author{
Mercedes Martín-Benito, 1, * Guillermo A. Mena Marugán, 1, 丹 and Edward Wilson-Ewing2, 田 \\ ${ }^{1}$ Instituto de Estructura de la Materia, \\ CSIC, Serrano 121, 28006 Madrid, Spain \\ ${ }^{2}$ Center for Fundamental Theory, Institute for Gravitation and the Cosmos, \\ Pennsylvania State University, University Park PA 16802, USA
}

We complete the quantization of the vacuum Bianchi I model within the framework of loop quantum cosmology adopting a new improved dynamics scheme put forward recently. In addition, we revisit the hybrid quantization of the Gowdy $T^{3}$ cosmologies with linear polarization using that scheme, proving with rigor some steps that remained unconcluded. The family of Gowdy $T^{3}$ cosmologies is an inhomogeneous model whose subset of homogeneous solutions is given precisely by the vacuum Bianchi I model. Our hybrid approach combines the new loop quantum cosmology description of this homogeneous sector with a Fock quantization of the inhomogeneities. Both in the Bianchi I model and in the Gowdy model the Hamiltonian constraint provides an evolution equation with respect to the volume of the Bianchi I universe, which is a discrete variable with a strictly positive minimum. We show that, in vacuo, this evolution is well defined inasmuch as the associated initial value problem is well posed: physical solutions are completely determined by the data on the initial section of constant Bianchi I volume. This fact allows us first to carry out to completion the quantization of the vacuum Bianchi I model which had not yet been achieved and then to confirm the feasibility of the hybrid procedure when the homogeneous sector is quantized with the new improved dynamics scheme.

PACS numbers: 4.60.Pp, 04.60.Kz, 98.80.Qc

\footnotetext{
* merce.martin@iem.cfmac.csic.es

$\dagger$ mena@iem.cfmac.csic.es

$\ddagger$ wilsonewing@gravity.psu.edu
} 


\section{INTRODUCTION}

In order to understand the dynamics of the very early universe, one must consider quantum gravity phenomena. With this aim, loop quantum cosmology (LQC) [1-3] confronts the quantization of symmetry reduced models in cosmology following loop quantum gravity (LQG) methods and ideas [4-6]. Although a full derivation of LQC from LQG has not yet been achieved, LQC is expected to correctly capture the behavior of the full theory, at least for those degrees of freedom which are responsible for the most important features of our universe.

Most of the work to date in LQC has been devoted to the study of the so-called minisuperspace models, which only contain a finite number of degrees of freedom. Despite their simplicity, these models are surprisingly rich inasmuch as they cover many of the situations and phenomena of interest in LQC and have already shed light on Planck-scale physics in a cosmological setting. The study of the flat homogeneous and isotropic cosmological model in LQC [7-13] has shown that the classical big bang singularity is resolved by quantum geometry effects and that a quantum bounce occurs when the matter energy density reaches the critical density $0.41 \rho_{\mathrm{Pl}}$. All this happens in such a way that the large volume limit of the model (for semiclassical states) is well approximated by the classical dynamics dictated by general relativity, just as one should expect. In addition, further studies have shown that the covariant entropy bound holds in this model [14] and that (assuming the presence of an inflaton field) the likelihood of obtaining viable initial conditions for slow roll inflation after the quantum bounce is higher than 99\% [15]. It has been proven as well that the quantum bounce persists and that the large volume limit gives the correct classical limit when a nonzero cosmological constant is present [16] and also for closed [17, 18] and open [19] topologies. Finally, although less is known about anisotropic models as their quantum dynamics are considerably more complex, it has been shown that the classical singularity is resolved both in the spatially flat Bianchi type I model [20-27] and the spatially curved Bianchi type II and type IX models [20, 28 31].

One of the key features in LQC is that the curvature is expressed in terms of holonomies and one must determine how these holonomies are to be constructed. For homogeneous and isotropic space-times, it took several years to understand that one should use the so-called $\bar{\mu}$-scheme or improved dynamics scheme, presented in Ref. [9], rather than the $\mu_{o}$-scheme or old dynamics scheme, which had been proposed in Ref. [8]. When anisotropic models were studied, there appeared two natural generalizations to the $\bar{\mu}$-scheme, which were presented in Ref. [23]. Since one of these was considerably simpler to work with, and a priori seemed more natural than the other, a lot of the initial LQC work studying Bianchi I models followed this procedure until several problems were pointed out [22, 26, 32]. In particular, the scaling properties of the (more complicated) alternate procedure have proven to be more suitable [24, 27]. Hence, unless one allows an explicit dependence of the $\bar{\mu}$-parameters on the coordinate cell adopted in the construction of the theory (or restricts the analysis exclusively to compact topologies where a distinguished choice of coordinate cell is available), the present consensus is that this alternate and more complicated procedure is the correct one to follow. We will refer to these two $\bar{\mu}$-schemes -i.e., the original simpler scheme and the more complicated one recently studied in Ref. [27] — as schemes A and B respectively.

The first aim of this paper is to complete the loop quantization of the vacuum Bianchi I model within scheme B, whose kinematical structure has been established in Ref. [27] and later analyzed in detail in Ref. [33]. We will see that the Hamiltonian constraint of the 
model provides a difference equation in an internal discrete parameter $v$, which is strictly positive and proportional to the volume of the Bianchi I universe. Employing the form of the superselection sectors for the anisotropies, which were determined in Ref. [33], we will show here that the quantum evolution equation is indeed well defined in vacuo, namely, that one can use $v$ as a "time" variable and evolve the wave function in terms of it. In other words, we will show that a set of initial data, given on the section of minimum $v$, completely determines the physical solutions. Owing to this fact, we will be able to obtain the physical structure of the vacuum Bianchi I model for the first time.

An extra motivation for the consideration of the vacuum Bianchi I model with the spatial topology of a three-torus is that its solutions coincide with the subset of homogeneous solutions (homogeneous sector) of the Gowdy $T^{3}$ model with linear polarization [34]. Based on the quantization of vacuum Bianchi I given here, one can then face the quantization of the Gowdy model in the framework of LQC, allowing for the introduction of inhomogeneities.

The Gowdy $T^{3}$ model with linear polarization can be viewed as the simplest inhomogeneous cosmological model. These cosmological spacetimes admit two axial Killing vector fields [34] and they describe universes devoid of matter which generically start with an initial curvature singularity [35, 36]. Their quantization by standard methods has been discussed in detail in the literature (see, e.g., Refs. [37, 38]). It is now well known that after a complete deparametrization, the model admits an essentially unique Fock quantization with certain desired properties 38]. This explains the attention that has already been paid to the quantization of the Gowdy $T^{3}$ spacetimes in the framework of the loop theory [39-42], with the two-fold purpose of including inhomogeneities in LQC and achieving the quantum resolution of the singularities of the model. In particular, Refs. [39, 40] succeeded in doing this by proposing a hybrid quantization which combines the loop quantization of the Bianchi type I homogeneous sector -where scheme A was adopted for the improved dynamics- with the natural Fock quantization of Ref. [38] for the inhomogeneities. Since it is generally accepted that scheme A must be replaced with the new scheme B in the quantization of the Bianchi I model, the second goal of this work is to discuss and complete the hybrid quantization of the Gowdy model making use of this alternate scheme for the homogeneous sector. With this aim, we will put on a rigorous basis some steps that are essential for the construction of a well-defined hybrid quantum model and which were left unfinished in Ref. [33].

Even though the kinematical Hilbert space of the hybrid procedure is naturally separated as the tensor product of two subspaces, namely the polymer space of the homogeneous sector times the Fock space for the inhomogeneities, the feasibility of this hybrid procedure is not at all trivial. As we will see, the Hamiltonian constraint of the model couples both sectors in a complicated way and it is not obvious whether the constraint can in fact be promoted to a well-defined operator, especially since the inhomogeneous sector contains an infinite number of degrees of freedom. Despite the complexity of the model, it was shown in Refs. [39, 40] that indeed the resulting hybrid quantization can be defined properly for scheme A and the corresponding physical Hilbert structure was obtained. Although a priori no relation of this structure with the kinematical one can be presumed, the standard quantum Fock description for the inhomogeneities was recovered in fact at the physical level. Now, the additional difficulties associated with scheme $\mathrm{B}$, where holonomies along different directions no longer commute, make the new problem considerably more complicated. In this paper, we will prove that the scheme B hybrid quantization of the Gowdy $T^{3}$ model is viable.

Let us mention that, apart from the analyses of the Gowdy model, other studies in the literature that have investigated the role of inhomogeneities in the framework of LQC are 
Ref. [43], which adopts a truncation of LQG and employs an approximation of the BornOppenheimer type, and the effective analysis of Ref. [44].

The paper is organized as follows. In Sec. II we recall and summarize the kinematical structure of the vacuum Bianchi I model with $T^{3}$ topology in LQC adopting scheme B. In Sec. III, first we prove that the notion of evolution with respect to the volume is well posed as the associated initial value problem is well defined, and then we complete the quantization, characterizing the physical Hilbert space and a(n over) complete set of physical observables. In Sec. IV, using the results obtained for the Bianchi I model, we show that the quantum dynamics of the Gowdy cosmology is also well defined when we employ scheme B in its hybrid quantization. Finally, we conclude in Sec. $[\mathrm{V}$ with a discussion of our results and further comments.

\section{BIANCHI I $T^{3}$ MODEL IN VACUO: KINEMATICS}

This section summarizes the kinematical structure of the Bianchi I model quantized adopting scheme $\mathrm{B}$ for the improved dynamics prescription. We also include the description of the superselection sectors. We refer the reader to Refs. [27, 33] for more details.

\section{A. Vacuum Bianchi I Hamiltonian Constraint}

In order to describe the classical model, we choose angular coordinates $\theta, \sigma, \delta \in S^{1}$ in which the spatial metric is diagonal. The elementary variables in loop quantum gravity are the Ashtekar-Barbero connection and the densitized triad. In the Bianchi I model, owing to homogeneity, each of them can be parametrized in a diagonal gauge by three coefficients. In terms of the fiducial co-triad $\{\mathrm{d} \theta, \mathrm{d} \sigma, \mathrm{d} \delta\}$ and the corresponding densitized fiducial triad, the coefficients of the Ashtekar-Barbero connection are given by $c_{i} /(2 \pi)$ and those of the densitized triad by $p_{i} /\left(4 \pi^{2}\right)$, with $i \in\{\theta, \sigma, \delta\}$ and where the denominators of $2 \pi$ come from the periods of our coordinates. These coefficients form three pairs of canonical variables, with

$$
\left\{c_{i}, p_{j}\right\}=8 \pi G \gamma \delta_{i j}
$$

Here, $\gamma$ is the Immirzi parameter, $G$ is Newton's constant, and $\delta_{i j}$ the Kronecker delta.

For the study of Bianchi I models in LQC using scheme B, it proves useful to introduce the variables

$$
\lambda_{i}=\frac{\operatorname{sgn}\left(p_{i}\right) \sqrt{\left|p_{i}\right|}}{(4 \pi \gamma \sqrt{\Delta})^{1 / 3} \ell_{\mathrm{Pl}}} \quad \text { and } \quad b_{i}=\sqrt{\left|\frac{p_{i} \Delta \ell_{\mathrm{Pl}}^{2}}{p_{j} p_{k}}\right|} c_{i},
$$

where $\ell_{\mathrm{Pl}}=\sqrt{G \hbar}$ is the Planck length while the area $\Delta \ell_{\mathrm{Pl}}^{2}$ is the gap in the spectrum of the area operator in LQG. In these equations, it is understood that repeated indices are not summed over and that the indices $i, j, k$ are all different. Note that the above change of variables is in fact well defined only for nonvanishing triad variables $p_{i}$. Later on, we will see that this suffices for the study of the kinematical arena in LQC.

It is also convenient to introduce the variable

$$
v=2 \lambda_{\theta} \lambda_{\sigma} \lambda_{\delta}
$$

which is proportional to the physical volume of the Bianchi I universe. 
The operators that appear in the Hamiltonian constraint of the Bianchi I model for the considered scheme (see Ref. [27] for details) are $\hat{\lambda}_{i}$ and $\widehat{\sin b_{i}}$ (or, equivalently, complex exponentials of $b_{i}$ ). It is easiest to work in the $\lambda_{i}$ representation, and in this case $\hat{\lambda}_{i}$ acts by multiplication while [45]

$$
\widehat{e^{i b_{\theta}}} \Psi\left(\lambda_{\theta}, \lambda_{\sigma}, \lambda_{\delta}\right)=\Psi\left(\lambda_{\theta}-\frac{1}{\left|\lambda_{\sigma} \lambda_{\delta}\right|}, \lambda_{\sigma}, \lambda_{\delta}\right),
$$

and likewise for the other complex exponentials of the $b_{i}$ 's. Finally, it is most convenient to change the configuration variables from $\left(\lambda_{\theta}, \lambda_{\sigma}, \lambda_{\delta}\right)$ to e.g. $\left(v, \lambda_{\sigma}, \lambda_{\delta}\right)$, since $v$ behaves in a simple manner under the action of $\widehat{e^{i b_{i}}}$, namely

$$
\begin{aligned}
& \widehat{e^{i b_{\theta}}} \Psi\left(v, \lambda_{\sigma}, \lambda_{\delta}\right)=\Psi\left(v-2 \cdot \operatorname{sgn}\left(\lambda_{\sigma} \lambda_{\delta}\right), \lambda_{\sigma}, \lambda_{\delta}\right), \\
& \widehat{e^{i b_{\sigma}}} \Psi\left(v, \lambda_{\sigma}, \lambda_{\delta}\right)=\Psi\left(v-2 \cdot \operatorname{sgn}\left(v \lambda_{\sigma}\right), \frac{v-2 \cdot \operatorname{sgn}\left(v \lambda_{\sigma}\right)}{v} \cdot \lambda_{\sigma}, \lambda_{\delta}\right),
\end{aligned}
$$

where the symbol sgn denotes the sign function. The action of $e^{\widehat{b_{\delta}}}$ is obtained by interchanging the roles of $\lambda_{\sigma}$ and $\lambda_{\delta}$ in the last equation. Thus, the effect of these holonomy operators on the dependence on $v$ is just a constant shift (up to a sign).

The Hamiltonian constraint operator obtained with a suitable choice of factor ordering and after a quantum densitization process [33] (such that the classical counterpart would appear in the action multiplied by the densitized lapse $N=N / \sqrt{\left|p_{\theta} p_{\sigma} p_{\delta}\right|}$ ) is given in scheme B by

$$
\hat{\mathcal{C}}_{H}=-\frac{1}{16 \pi G \gamma^{2}}\left[\hat{\Theta}_{\theta} \hat{\Theta}_{\sigma}+\hat{\Theta}_{\sigma} \hat{\Theta}_{\theta}+\hat{\Theta}_{\theta} \hat{\Theta}_{\delta}+\hat{\Theta}_{\delta} \hat{\Theta}_{\theta}+\hat{\Theta}_{\sigma} \hat{\Theta}_{\delta}+\hat{\Theta}_{\delta} \hat{\Theta}_{\sigma}\right],
$$

where

$$
\hat{\Theta}_{i}=\pi \gamma \ell_{\mathrm{Pl}}^{2} \widehat{\sqrt{|v|}}\left[\widehat{\sin \left(b_{i}\right)} \widehat{\operatorname{sgn}\left(\lambda_{i}\right)}+\widehat{\operatorname{sgn}\left(\lambda_{i}\right)} \widehat{\sin \left(b_{i}\right)}\right] \widehat{\sqrt{|v|}} .
$$

Note that this is a different factor ordering choice for $\hat{\Theta}_{i}$ than what was chosen in Ref. [27]. This choice is preferable because the operator has the same action on wave functions supported at large values of $\lambda_{i}$ while its action for small values of $\lambda_{i}$ is much simpler and the consequences are more transparent. Most importantly, the action of this operator does not allow any communication between different $\left(\lambda_{\theta}, \lambda_{\sigma}, \lambda_{\delta}\right)$ octants [25, 33, 39].

Under the action of $\hat{\mathcal{C}}_{H}$, the zero volume states decouple. Therefore, the singularities are resolved kinematically inasmuch as the quantum states that would correspond to them can be removed in practice from the kinematical Hilbert space [25, 33, 39]. Besides, under the action of this Hamiltonian constraint, the different octants remain invariant. Then, each octant contains different superselection sectors. This and the fact that the octants are all related by parity allow us to restrict our attention to the (strictly) positive octant. We do this for the remainder of this paper. Then, the Hamiltonian constraint acting on a wave function where $v>4$ gives

$$
\begin{aligned}
0= & \sqrt{v}\left[(v+2) \sqrt{v+4} \Psi_{4}^{+}\left(v+4, \lambda_{\sigma}, \lambda_{\delta}\right)-(v+2) \sqrt{v} \Psi_{0}^{+}\left(v, \lambda_{\sigma}, \lambda_{\delta}\right)\right. \\
& \left.-(v-2) \sqrt{v} \Psi_{0}^{-}\left(v, \lambda_{\sigma}, \lambda_{\delta}\right)+(v-2) \sqrt{v-4} \Psi_{4}^{-}\left(v-4, \lambda_{\sigma}, \lambda_{\sigma}\right)\right] .
\end{aligned}
$$


Here $\Psi_{0,4}^{ \pm}$are defined as follows:

$$
\begin{aligned}
\Psi_{n}^{ \pm}\left(v \pm n, \lambda_{\sigma}, \lambda_{\delta}\right)= & \Psi\left(v \pm n, \frac{v \pm n}{v \pm 2} \cdot \lambda_{\sigma}, \frac{v \pm 2}{v} \cdot \lambda_{\delta}\right)+\Psi\left(v \pm n, \frac{v \pm n}{v \pm 2} \cdot \lambda_{\sigma}, \lambda_{\delta}\right) \\
& +\Psi\left(v \pm n, \frac{v \pm 2}{v} \cdot \lambda_{\sigma}, \frac{v \pm n}{v \pm 2} \cdot \lambda_{\delta}\right)+\Psi\left(v \pm n, \frac{v \pm 2}{v} \cdot \lambda_{\sigma}, \lambda_{\delta}\right) \\
& +\Psi\left(v \pm n, \lambda_{\sigma}, \frac{v \pm 2}{v} \cdot \lambda_{\delta}\right)+\Psi\left(v \pm n, \lambda_{\sigma}, \frac{v \pm n}{v \pm 2} \cdot \lambda_{\delta}\right)
\end{aligned}
$$

On the other hand, if $2<v \leq 4$, the contribution of $\Psi_{4}^{-}$disappears in Eq. (2.9), whereas if $0<v \leq 2$ the two last contributions in that equation, namely those proportional to $\Psi_{4}^{-}$ and $\Psi_{0}^{-}$, are absent.

\section{B. Superselection Sectors}

It was already pointed out in Ref. [27] that there are superselection sectors in $v$, denoted by a continuous parameter $\epsilon \in(0,4]$. Given such a superselection sector, wave functions only have support on points where $v=\epsilon+4 n, n$ being a natural number.

Remarkably, there are also superselection sectors in the $\lambda_{i}$ 's, although these sectors have a quite different structure compared to those in $v$. As it is shown in Ref. [33], given $\epsilon$ and an initial value $\lambda_{i}^{\star}>0$, the wave function will only have support on those points that can be expressed in the form

$$
\lambda_{i}=\left(\frac{\epsilon-2}{\epsilon}\right)^{z} \prod_{k}\left(\frac{\epsilon+2 m_{k}}{\epsilon+2 n_{k}}\right)^{p_{k}} \lambda_{i}^{\star},
$$

for some $k$, where $m_{k}, n_{k}$, and $p_{k}$ are nonnegative integers, and $z$ is any integer unless $\epsilon \leq 2$, in which case $z=0$. Note that different $\lambda_{i}^{\star}$ s will yield the same superselection sector if they are related by Eq. (2.11). It is not difficult to see that one of the available superselection sectors is the set of nonnegative rational numbers (it suffices to consider the case $\epsilon=2$ with $\lambda_{i}^{\star}$ being a rational number). Furthermore, it follows from Eq. (2.11) that each superselection sector is countable, dense in $\mathbb{R}^{+}$, and that all superselection sectors are isomorphic [33].

Finally, one can show that the form of the superselection sectors for the three wave function variables $\left(v, \lambda_{\sigma}, \lambda_{\delta}\right)$ is characterized by three numbers $\left(\epsilon, \lambda_{\sigma}^{\star}, \lambda_{\delta}^{\star}\right)$, and is given by the tensor product of the superselection sectors for each individual variable, that is to say that there is no restriction on the sector of $\lambda_{\delta}$ given that of $\lambda_{\sigma}$ or vice versa [33]. In particular, note that if $\lambda_{\sigma}^{\star}$ and $\lambda_{\delta}^{\star}$ are compatible in the sense of Eq. (2.11), then the superselection sectors of $\lambda_{\sigma}$ and $\lambda_{\delta}$ are the same. It is clear that, given one superselection sector $\left(\epsilon, \lambda_{\sigma}^{\star}, \lambda_{\delta}^{\star}\right)$, a wave function will only have support on a countable number of points. Now, whereas the superselection sector in $v$ only contains information about a discrete set of points separated by a constant shift, the superselection sector in the $\lambda_{i}$ 's encodes the information of a set of points which are densely distributed in the positive quadrant.

\section{BIANCHI I $T^{3}$ MODEL IN VACUO: PHYSICAL STRUCTURE}

In this section we will analyze the solutions to the Hamiltonian constraint. We see from Eq. (2.9) that the constraint provides a difference equation in the parameter $v$, and thus 
it can be regarded as a (discrete) evolution equation in this parameter. In the previous analysis of Bianchi I carried out in Ref. [27], a matter content was added, and the notion of evolution was developed in terms of a massless scalar field, instead of doing it in terms of this volume parameter $v$, which has a purely geometric nature. In the former case, thanks to the suitable properties of the massless field, which is quantized in a standard Schrödinger-like representation, it is straightforward to prove that the associated initial value problem is well posed. In fact, in this respect the situation is quite similar to that found in (relativistic) Quantum Mechanics. Nonetheless, regarding the geometry part, the physical structure of the solutions remained unanswered. Now, in the vacuum case considered here, the role of "time" is played by the volume variable $v$, which has been polymerically quantized. Because of its discrete nature, the fact that the associated notion of evolution is well defined is not trivial. We will show in this section that the dynamics are correctly posed: a set of initial data evaluated on the section of initial $v$ completely determines the physical solution. As we will see, the proof is not direct, owing to the complexity of scheme B. In turn, this result will allow us to obtain for the first time the physical Hilbert space of the vacuum Bianchi I model in LQC and a(n over) complete set of observables, thus completing the quantization of the model.

\section{A. Solutions to the Hamiltonian Constraint}

Since we do not expect generic solutions to the Hamiltonian constraint to be normalizable in the kinematical Hilbert space, we will look for solutions in a larger space, namely the algebraic dual of a suitable domain of definition for the Hamiltonian constraint operator. It will be convenient to work with the variables $x_{i}=\ln \lambda_{i}$ instead of the $\lambda_{i}$ 's themselves, as the former variables run over the real line while the latter are positive and, besides, the $x_{i}$ 's suffer displacements under the action of the Hamiltonian constraint operator instead of dilatations or contractions. In fact, from Eq. (2.11), the superselection sectors in $x_{i}$ are formed by those points such that $\left(x_{i}-\ln \lambda_{i}^{\star}\right)=w_{i} \in \mathcal{Z}_{\epsilon}$, where

$$
\mathcal{Z}_{\epsilon}=\left\{z \ln \left(\frac{\epsilon-2}{\epsilon}\right)+\sum_{k} \bar{p}_{k} \ln \left(\frac{\epsilon+2 \bar{m}_{k}}{\epsilon+2 \bar{n}_{k}}\right)\right\} .
$$

Here, for convenience, we have slightly changed the notation with respect to Eq. (2.11), so that now $\bar{m}_{k} \geq \bar{n}_{k}$ are nonnegative integers, and $\bar{p}_{k}$ can take any integer value. Recall that $z$ is any integer unless $\epsilon \leq 2$ in which case $z=0$. We note that $\mathcal{Z}_{\epsilon}$ is dense in the real line, because the superselection sectors of $\lambda_{i}$ are dense in $\mathbb{R}^{+}$and the logarithm is a continuous function from the positive axis to the real line. In spite of the introduction of the $x_{i}$ 's, we will still keep $v$ as one of our variables given its nice behavior under the action of the Hamiltonian constraint operator.

Since the Wheeler-DeWitt equation associated to the Bianchi I model is actually a first order differential equation in the three variables $x_{i}$, it should be possible to determine the entire solution to the Hamiltonian constraint supplying as initial data its restriction to one Cauchy slice (i.e., a surface with constant value of one of the $x_{i}$ 's or alternatively with constant $v$ ). However, the constraint in LQC is a second order difference operator for generic values of $v$. Therefore, it is not immediately clear how the solution can be determined from one slice of initial data obtained at a constant value of $v$. The solution lies in the different 
form that the action of the Hamiltonian constraint operator has on states with $v \leq 4$, this form is that of a first order difference equation.

Given a superselection sector in $v$, denoted by $\epsilon \in(0,4]$, one obtains this first order difference equation in $v$ for $v=\epsilon+4$ in terms of the initial data on the slice $v=\epsilon$. If one can solve this (highly coupled) difference equation, it will then be possible to solve the (again highly coupled) second order difference equation for $v=\epsilon+8$ in terms of the data on the slices $v=\epsilon$ and $v=\epsilon+4$. One can then follow this strategy in order to obtain the full solution to the Hamiltonian constraint for all $v$. Finally, because the $v+4$ terms always appear in the same combination, given by $\Psi_{4}^{+}$, we only need to show how to derive the data on $v=\epsilon+4$, and then the data for all other $v$ can be obtained in the same manner.

The difference equation we are interested in is

$$
\Psi_{4}^{+}\left(\epsilon+4, x_{\sigma}, x_{\delta}\right)=\sqrt{\frac{\epsilon}{\epsilon+4}}\left[\Psi_{0}^{+}\left(\epsilon, x_{\sigma}, x_{\delta}\right)+\frac{\epsilon-2}{\epsilon+2} \Psi_{0}^{-}\left(\epsilon, x_{\sigma}, x_{\delta}\right)\right],
$$

where the second term on the righthand side is absent if $\epsilon \leq 2$. Since the righthand side is known, the question is just whether one is able to obtain $\Psi\left(\epsilon+4, x_{\sigma}, x_{\delta}\right)$ from $\Psi_{4}^{+}\left(\epsilon+4, x_{\sigma}, x_{\delta}\right)$ in order to derive the form of the wave function for all $v$.

The explicit form of $\Psi_{4}^{+}$is given by:

$$
\begin{aligned}
\Psi_{4}^{+}\left(v+4, x_{\sigma}, x_{\delta}\right) & =\Psi\left(v+4, x_{\sigma}, \ln \left(\frac{v+4}{v+2}\right)+x_{\delta}\right)+\Psi\left(v+4, \ln \left(\frac{v+4}{v+2}\right)+x_{\sigma}, x_{\delta}\right) \\
& +\Psi\left(v+4, x_{\sigma}, \ln \left(\frac{v+2}{v}\right)+x_{\delta}\right)+\Psi\left(v+4, \ln \left(\frac{v+2}{v}\right)+x_{\sigma}, x_{\delta}\right) \\
& +\Psi\left(v+4, \ln \left(\frac{v+2}{v}\right)+x_{\sigma}, \ln \left(\frac{v+4}{v+2}\right)+x_{\delta}\right) \\
& +\Psi\left(v+4, \ln \left(\frac{v+4}{v+2}\right)+x_{\sigma}, \ln \left(\frac{v+2}{v}\right)+x_{\delta}\right)
\end{aligned}
$$

for any value of $v$ (and, in particular, for $v=\epsilon$ ). It can be expressed as the result of the action of two separate operators,

$$
\Psi_{4}^{+}\left(v+4, x_{\sigma}, x_{\delta}\right)=\hat{U}_{6}(v+4) \hat{A} \Psi\left(v, x_{\sigma}, x_{\delta}\right),
$$

where $\hat{A}$ only shifts the value of $v$, namely,

$$
\hat{A} \Psi\left(v, x_{\sigma}, x_{\delta}\right)=\left(\widehat{e^{-i b_{\theta}}}\right)^{2} \Psi\left(v, x_{\sigma}, x_{\delta}\right)=\Psi\left(v+4, x_{\sigma}, x_{\delta}\right)
$$

while

$$
\begin{aligned}
\hat{U}_{6}(v) \Psi\left(v, x_{\sigma}, x_{\delta}\right)= & {\left[\widehat{e^{-i b_{\theta}}} \widehat{e^{-i b_{\sigma}}}+\widehat{e^{-i b_{\sigma}}} \widehat{e^{-i b_{\theta}}}+\widehat{e^{-i b_{\sigma}}} \widehat{e^{-i b_{\delta}}}+\widehat{e^{-i b_{\delta}}} \widehat{e^{-i b_{\sigma}}}\right.} \\
& \left.+\widehat{e^{-i b_{\delta}}} \widehat{e^{-i b_{\theta}}}+\widehat{e^{-i b_{\theta}}} \widehat{e^{-i b_{\delta}}}\right]\left(\widehat{e^{i b_{\theta}}}\right)^{2} \Psi\left(v, x_{\sigma}, x_{\delta}\right)
\end{aligned}
$$

has a trivial action on the $v$-sector.

The invertibility of the operator $\hat{U}_{6}(v)$ for any value of $v$ would guarantee that one can determine $\Psi\left(v, x_{\sigma}, x_{\delta}\right)$ from $\Psi_{4}^{+}\left(v, x_{\sigma}, x_{\delta}\right)$. Assuming for the moment that the inverse operator $\left[\hat{U}_{6}(v)\right]^{-1}$ exists, we can derive the state at the volume $\epsilon+4$ by calculating

$$
\Psi\left(\epsilon+4, x_{\sigma}, x_{\delta}\right)=\sqrt{\frac{\epsilon}{\epsilon+4}}\left[\hat{U}_{6}(\epsilon+4)\right]^{-1}\left[\Psi_{0}^{+}\left(\epsilon, x_{\sigma}, x_{\delta}\right)+\frac{\epsilon-2}{\epsilon+2} \Psi_{0}^{-}\left(\epsilon, x_{\sigma}, x_{\delta}\right)\right] .
$$


Once again, the second term in the square brackets on the right hand side of this equation does not appear if $\epsilon \leq 2$.

Again assuming the existence of $\left[\hat{U}_{6}(v)\right]^{-1}$, it is now straightforward to obtain the value of the wave function for the section $v=\epsilon+8$ :

$$
\begin{aligned}
\Psi\left(\epsilon+8, x_{\sigma}, x_{\delta}\right)= & \sqrt{\frac{\epsilon+4}{\epsilon+8}}\left[\hat{U}_{6}(\epsilon+8)\right]^{-1}\left[\Psi_{0}^{+}\left(\epsilon+4, x_{\sigma}, x_{\delta}\right)+\frac{\epsilon+2}{\epsilon+6} \Psi_{0}^{-}\left(\epsilon+4, x_{\sigma}, x_{\delta}\right)\right. \\
& \left.-\frac{\epsilon+2}{\epsilon+6} \sqrt{\frac{\epsilon}{\epsilon+4}} \Psi_{4}^{-}\left(\epsilon, x_{\sigma}, x_{\delta}\right)\right] .
\end{aligned}
$$

It is clear how to repeat this procedure in order to get the value of the wave function for all larger $v$ as well. Therefore, if the operator $\hat{U}_{6}(v)$ can be inverted we conclude that the initial value problem in terms of $v$ is well posed, at least at $v=\epsilon$. In the next section we show that indeed this is the case.

\section{B. The operator $\hat{U}_{6}$}

Let us then analyze the operator $\hat{U}_{6}(v)$ to see that its action can be inverted. First, we provide a suitable domain of definition for $\hat{U}_{6}(v)$, keeping fixed the value of $v$ (in other words, we restrict the discussion just to a slice of constant $v$ ). For each direction $i=\sigma$ or $\delta$, consider the linear span $\mathrm{Cyl}_{\lambda_{i}^{\star}}$ of the states whose support is just one point $x_{i}$ of the superselection sector determined by Eq. (3.1), with $\left(x_{i}-\ln \lambda_{i}{ }^{\star}\right)=w_{i} \in \mathcal{Z}_{\epsilon}$. We call $\mathcal{H}_{\lambda_{i}^{\star}}$ the Hilbert completion of this vector space with the discrete inner product. Then, we can choose the tensor product $\mathrm{Cyl}_{\lambda_{\sigma}^{\star}} \otimes \mathrm{Cyl}_{\lambda_{\delta}^{\star}}$ as the domain for $\hat{U}_{6}(v)$.

Now, if we define on the Hilbert space $\mathcal{H}_{\lambda_{\sigma}^{\star}} \otimes \mathcal{H}_{\lambda_{\delta}^{\star}}$ the translations

$$
\hat{U}^{\left(w_{\sigma}, w_{\delta}\right)} \Psi\left(v, x_{\sigma}, x_{\delta}\right)=\Psi\left(v, w_{\sigma}+x_{\sigma}, w_{\delta}+x_{\delta}\right),
$$

then the operator $\hat{U}_{6}(v)$ is just a sum of six translations of this kind. These translation operators are unitary because, if $w_{\sigma}$ and $w_{\delta}$ are two numbers in $\mathcal{Z}_{\epsilon}$, so that a shift of $x_{i}$ by any of them leaves invariant the superselection sector [see Eq. (3.1)], then the sum of $\left|\hat{U}^{\left(w_{\sigma}, w_{\delta}\right)} \Psi\left(v, x_{\sigma}, x_{\delta}\right)\right|^{2}$ over all $x_{\sigma}$ and $x_{\delta}$ in the superselection sector coincides with the sum of $\left|\Psi\left(v, x_{\sigma}, x_{\delta}\right)\right|^{2}$. Moreover, owing to this property and the Schwarz inequality, we conclude that the norm of the operator $\hat{U}_{6}(v)$ is bounded by 6 .

Since $\hat{U}_{6}(v)$ is bounded, it can be extended as a well-defined operator to the entire Hilbert space. This extension [which we also denote by $\hat{U}_{6}(v)$ ] provides in fact a normal operator - namely, the operator commutes with its adjoint - as the translations in $x_{\sigma}$ and/or in $x_{\delta}$ commute. Hence, in particular, it is guaranteed that the residual spectrum is empty. Thus the operator $\hat{U}_{6}(v)$ is invertible in our Hilbert space if and only if its point spectrum does not contain the zero.

It is not difficult to convince oneself that the point spectrum of $\hat{U}_{6}(v)$ must be empty owing to the properties of the operator. The idea is that since this operator is just a linear combination of translations, any of its eigenfunctions must possess a certain translational invariance which would prevent them from being normalizable. In order to see this, let us consider again the translations $\hat{U}^{\left(w_{\sigma}, w_{\delta}\right)}$, with $w_{\sigma}, w_{\delta} \in \mathcal{Z}_{\epsilon}$. Since they all commute with 
each other as well as with $\hat{U}_{6}(v)$, they can all be diagonalized simultaneously, that is, there exists a basis of common (generalized) eigenfunctions. Let us call in the following

$$
\hat{U}_{\sigma}^{w_{\sigma}}=\hat{U}^{\left(w_{\sigma}, 0\right)}, \quad \hat{U}_{\delta}^{w_{\delta}}=\hat{U}^{\left(0, w_{\delta}\right)},
$$

so that $\hat{U}^{\left(w_{\sigma}, w_{\delta}\right)}=\hat{U}_{\sigma}^{w_{\sigma}} \hat{U}_{\delta}^{w_{\delta}}$. Given one of the eigenfunctions common to all of these translations, we denote the corresponding eigenvalue of $\hat{U}_{i}^{w_{i}}$ by $\rho_{i}\left(w_{i}\right)$ (with $i=\sigma, \delta$ ). This eigenvalue must be a complex number of unit norm, because the translation operators are unitary. In addition, since $\hat{U}_{i}^{w_{i}} \hat{U}_{i}^{\bar{w}_{i}}=\hat{U}_{i}^{w_{i}+\bar{w}_{i}}$, it follows that

$$
\rho_{i}\left(w_{i}\right) \rho_{i}\left(\bar{w}_{i}\right)=\rho_{i}\left(w_{i}+\bar{w}_{i}\right) .
$$

Recalling that all points in the superselection sector can be reached from $\ln \lambda_{i}^{\star}$ by a translation $\hat{U}_{i}^{w_{i}}$, it is a simple exercise to show that the eigenfunctions are proportional to $\rho_{\sigma}\left(w_{\sigma}\right) \rho_{\delta}\left(w_{\delta}\right)$. We can always change this wave function by a constant of unit norm, and thus we fix $\rho_{i}(0)=1$. Besides, in order to determine completely the wave function, we only need to know the value of $\rho_{i}\left(w_{i}\right)$ in an appropriate subset of $\mathcal{Z}_{\epsilon}$, namely any collection of noncommensurable points which can generate the entire set by multiplication by integers. It is possible to see that property (3.11) provides then all the information about $\rho_{i}$ at the rest of points in $\mathcal{Z}_{\epsilon}$. In particular, $\rho_{i}\left(n w_{i}\right)=\left[\rho_{i}\left(w_{i}\right)\right]^{n}$.

The wave functions $\rho_{i}\left(w_{i}\right)$ are clearly nonnormalizable with respect to the discrete inner product in $\mathcal{H}_{\lambda_{i}^{\star}}$ as they have complex unit norm at each point of the superselection sector (the shift of $\mathcal{Z}_{\epsilon}^{i}$ by $\ln \lambda_{i}^{\star}$ ) and the sector contains an infinite number of points. In addition, different wave functions $\rho_{i}\left(w_{i}\right)$ must be orthogonal, because there always exists a (unitary) translation operator on $\mathcal{H}_{\lambda_{i}^{\star}}$ whose eigenvalue differs for the two wave functions.

At this stage of the discussion, it is worth noticing that, by the very construction of the algebra of fundamental operators in LQC previous to the introduction of superselection sectors, the operators $\hat{U}_{i}^{w_{i}}$ that act as translations in the $x_{i}$ representation can be identified in the holonomy/connection representation - where they act as multiplicative operatorsas elements of the Bohr compactification of the real line, $\mathbb{R}_{\text {Bohr }}$ [46]. These elements can be understood as maps $\rho_{i}$ from the real line (corresponding to all possible real values of $x_{i}$, or equivalently of $\left.w_{i}\right)$ to the circle such that they satisfy condition (3.11) and $\rho_{i}(0)=1$. Owing to superselection, however, the values of $w_{i}$ are now restricted to belong to $\mathcal{Z}_{\epsilon}$. We can then identify the wave functions $\rho_{i}\left(w_{i}\right)$ as equivalence classes of elements in $\mathbb{R}_{\text {Bohr }}$, the equivalence relation being the identification of all those maps $\rho_{i}$ which differ only by their action on the set complementary to $\mathcal{Z}_{\epsilon}$ in the real line, i.e., $\mathbb{R} \backslash \mathcal{Z}_{\epsilon}$. Examples of $\rho_{i}\left(w_{i}\right)$ are provided by the exponential maps $\exp \left(i k_{i} w_{i}\right)$ from $\mathcal{Z}_{\epsilon}$ to $S^{1}$. Since $\mathcal{Z}_{\epsilon}$ contains noncommensurable numbers, these exponentials separate all real values of $k_{i}$ [that is, for any two values of $k_{i}$ one can find a value of $w_{i}$ for which the exponentials $\exp \left(i k_{i} w_{i}\right)$ are different]. So, the set of possible and distinct $\rho_{i}$ contains all the exponentials with $k_{i} \in \mathbb{R}$.

Returning to the operator $\hat{U}_{6}(v)$, it is straightforward to find its eigenvalue for each of the analyzed wave functions. It is given by

$$
\begin{aligned}
\omega_{6}\left(\rho_{\sigma}, \rho_{\delta}\right) & =\sum_{i=\sigma, \delta}\left\{\rho_{i}\left[\ln \left(\frac{v}{v-2}\right)\right]+\rho_{i}\left[\ln \left(\frac{v-2}{v-4}\right)\right]\right\} \\
& +\sum_{i, j=\sigma, \delta ; i \neq j}\left\{\rho_{i}\left[\ln \left(\frac{v}{v-2}\right)\right] \rho_{j}\left[\ln \left(\frac{v-2}{v-4}\right)\right]\right\} .
\end{aligned}
$$


Remember that here $v>4$. The point spectrum of $\hat{U}_{6}(v)$ will not contain the zero provided that there is no normalizable linear superposition of the above wave functions with $\omega_{6}\left(\rho_{\sigma}, \rho_{\delta}\right)=0$. In this superposition, the measure for $\rho_{i}$ is continuous: this is a consequence of the wave functions $\rho_{i}\left(w_{i}\right)$ not being normalizable in $\mathcal{H}_{\lambda_{i}^{\star}}$. The restriction to the kernel of $\hat{U}_{6}(v)$ is achieved then by introducing a delta function of $\omega_{6}\left(\rho_{\sigma}, \rho_{\delta}\right)$ (peaked at zero). If one computes the norm of this superposition, the orthogonality of the wave functions $\rho_{i}\left(w_{i}\right)$ leads to integrals over the square complex norm of each $\left(\rho_{\sigma}, \rho_{\delta}\right)$-contribution. But this contains a square delta, so that the norm diverges. Therefore, the point spectrum of the operator $\hat{U}_{6}(v)$ does not contain the zero, as we wanted to show. Actually, one can apply the same line of reasoning for any other possible eigenvalue of $\hat{U}_{6}$, not just for zero, showing that in fact the point spectrum of this operator is empty.

\section{Physical Hilbert space}

Now that we have seen that the solutions to the Hamiltonian constraint are completely determined by the data on the initial slice $v=\epsilon$, we can identify these solutions with the corresponding initial data and characterize the physical Hilbert space by providing a Hilbert structure to the data, belonging in principle to the dual of the vector space $\mathrm{Cyl}_{\lambda_{\sigma}^{\star}} \otimes \mathrm{Cyl}_{\lambda_{\delta}^{\star}} \subset$ $\mathcal{H}_{\lambda_{\sigma}^{\star}} \otimes \mathcal{H}_{\lambda_{\delta}^{\star}}$.

In order to endow them with an inner product, we take a(n over) complete set of classical observables forming a closed algebra, and we impose that the quantum counterpart of their complex conjugation relations become adjointness relations between operators. Such a set is formed by the operators $\widehat{e^{i x_{i}}}$ and $\hat{U}_{i}^{\omega_{i}}$, with $\omega_{i} \in \mathcal{Z}_{\epsilon}$ and $i=\sigma, \delta$. For $\psi\left(\lambda_{\sigma}, \lambda_{\delta}\right) \in \mathrm{Cyl}_{\lambda_{\sigma}^{\star}} \otimes \mathrm{Cyl}_{\lambda_{\delta}^{\star}}$ (and for the initial data by duality), these operators are defined as

$$
\begin{aligned}
\widehat{e^{i x_{\sigma}}} \psi\left(x_{\sigma}, x_{\delta}\right) & =e^{i x_{\sigma}} \psi\left(x_{\sigma}, x_{\delta}\right), \\
\hat{U}_{\sigma}^{\omega_{\sigma}} \psi\left(x_{\sigma}, x_{\delta}\right) & =\psi\left(x_{\sigma}+\omega_{\sigma}, x_{\delta}\right),
\end{aligned}
$$

and similarly for $\widehat{e^{i x_{\delta}}}$ and $\hat{U}_{\delta}^{\omega_{\delta}}$. Clearly, all these operators are unitary in $\mathcal{H}_{\lambda_{\sigma}^{\star}} \otimes \mathcal{H}_{\lambda_{\delta}^{\star}}$, according with their reality conditions. Therefore, we conclude that this Hilbert space is precisely the physical Hilbert space of the vacuum Bianchi I model.

\section{HYBRID QUANTIZATION OF THE GOWDY $T^{3}$ COSMOLOGIES}

The Gowdy $T^{3}$ model can be viewed as homogeneous Bianchi I backgrounds which allow certain inhomogeneous modes of the gravitational field to propagate along one direction. This natural separation in homogeneous and inhomogeneous sectors motivated a hybrid quantization of the model which combines the loop quantization of the Bianchi I phase space with a natural Fock quantization for the inhomogeneities, and which was carried out in Refs. [39, 40] adopting scheme A for the improved dynamics in the quantization of the Bianchi I sector. This separation of degrees of freedom is nonperturbative and independent of the strength of the inhomogeneities at the classical level. Although ideally one should perform a LQC quantization for the inhomogeneous degrees of freedom as well, this hybrid approach is justified if the most relevant quantum geometry effects (but not necessarily all quantum effects) are those that affect the homogenous background so that one can establish 
a kind of perturbative hierarchy in their treatment. In addition, it is natural to adopt a Fock quantization of the inhomogeneities in this context based on the expectation that a conventional Fock description of the inhomogeneities ought to be recovered from LQC in a regime where quantum geometry phenomena are negligible. In this case there exists a privileged Fock quantization under certain requirements on the symmetries of the vacuum and on the existence of a unitary dynamics with respect to an emergent time [38]; these properties provide a natural Fock quantization for the inhomogeneous modes.

Here, we will show that the hybrid quantization of the Gowdy model employing scheme $\mathrm{B}$ for the loop quantization of the homogeneous sector is also viable. As in previous works with the other scheme [39, 40], this is not a trivial issue owing to the coupling between the homogeneous and inhomogeneous sectors in the Hamiltonian constraint. Moreover, now the structure of the homogeneous sector is much more complicated, owing to the intricacy of the holonomy operators in the new scheme B. The results obtained in Sec. III will be essential in order to see that the hybrid quantization is well defined within scheme B as well. Our demonstration provides a necessary justification for the steps followed in Ref. [33] where the physical Hilbert space of this new hybrid Gowdy model was obtained.

\section{A. Kinematical structure and Hamiltonian constraint operator}

As in the Bianchi I model, since the spatial topology is that of a three-torus, we have $\theta, \sigma, \delta \in S^{1}$ with a coordinate length of $2 \pi$. Following a careful gauge-fixing [40, 47], one finds that the information about the homogeneous degrees of freedom (which describe the subfamily of homogeneous space-times in the Gowdy model) can be encoded in the Bianchi I variables $c_{i}$ and $p_{i}$ introduced in Sec. IIA. On the other hand, the inhomogeneities corresponding to the content of gravitational waves can be described by a single metric field (without a zero mode), which in turn can be described by creation and annihilation-like variables $\left\{\left(a_{m}, a_{m}^{*}\right), m \in \mathbb{Z}-\{0\}\right\}$, defined in the same way as the natural variables that one would adopt if the field behaved as a free massless scalar field. Owing to the partial gauge-fixing, only two global constraints remain on the system: the zero mode of the Hamiltonian constraint, which generates time reparametrizations, and the zero mode of the $\theta$-diffeomorphism constraint, which generates translations around the $\theta$-circle (see Ref. [40] for details).

In order to proceed with the hybrid quantization of the Gowdy model, we follow the LQC approach for the homogeneous degrees of freedom and, as in the Bianchi I model, we adopt the prescription $p_{i} c_{i} \rightarrow \hat{\Theta}_{i}$ where the operators $\hat{\Theta}_{i}$ are defined in Eq. (2.8) while for the inhomogeneities we promote the creation and annihilation variables to operators in the standard quantum field theory fashion. The kinematical Hilbert space is then the tensor product of the polymer space of the Bianchi I model times the resulting Fock space for the inhomogeneities [33].

The generator of translations around the $\theta$-circle only affects the inhomogeneities and it is straightforward to impose in the quantum theory [33, 39, 40]. On the other hand, as we pointed out earlier, the Hamiltonian constraint couples the homogeneous and inhomogeneous sectors in a nontrivial way. The resulting operator has the explicit form [33] 


$$
\begin{aligned}
\hat{\mathcal{C}}_{H}=- & \frac{1}{16 \pi G \gamma^{2}}\left[\hat{\Theta}_{\theta} \hat{\Theta}_{\sigma}+\hat{\Theta}_{\sigma} \hat{\Theta}_{\theta}+\hat{\Theta}_{\theta} \hat{\Theta}_{\delta}+\hat{\Theta}_{\delta} \hat{\Theta}_{\theta}+\hat{\Theta}_{\sigma} \hat{\Theta}_{\delta}+\hat{\Theta}_{\delta} \hat{\Theta}_{\sigma}\right] \\
& +\frac{1}{16 \pi}\left(\left[\overline{\frac{1}{\left|p_{\theta}\right|^{1 / 4}}}\right]^{2} \frac{\left(\hat{\Theta}_{\sigma}+\hat{\Theta}_{\delta}\right)^{2}}{\gamma^{2}}\left[\widehat{\frac{1}{\left|p_{\theta}\right|^{1 / 4}}}\right]^{2} \widehat{\mathrm{H}_{\mathrm{int}}^{\xi}}+32 \pi^{2} \mid \widehat{p_{\theta} \mid} \widehat{\mathrm{H}_{o}^{\xi}}\right)
\end{aligned}
$$

where

$$
\widehat{\mathrm{H}_{\mathrm{o}}^{\xi}}=\sum_{m \neq 0}|m| \hat{a}_{m}^{\dagger} \hat{a}_{m} \quad \text { and } \quad \widehat{\mathrm{H}_{\mathrm{int}}^{\xi}}=\sum_{m \neq 0} \frac{1}{2|m|}\left(2 \hat{a}_{m}^{\dagger} \hat{a}_{m}+\hat{a}_{m} \hat{a}_{-m}+\hat{a}_{m}^{\dagger} \hat{a}_{-m}^{\dagger}\right)
$$

are the terms that involve the inhomogeneities and the regulated "inverse triad" operator representing $\left|p_{\theta}\right|^{-1 / 4}$ is given by [29, 33]

$$
\left[\overline{\frac{1}{\left|p_{\theta}\right|^{1 / 4}}}\right]\left|v, \lambda_{\sigma}, \lambda_{\delta}\right\rangle=\frac{\sqrt{2\left|\lambda_{\sigma} \lambda_{\delta}\right|}}{\left(4 \pi \gamma \sqrt{\Delta} \ell_{\mathrm{Pl}}^{3}\right)^{1 / 6}}(\sqrt{|v+1|}-\sqrt{|v-1|})\left|v, \lambda_{\sigma}, \lambda_{\delta}\right\rangle .
$$

Note that the first term in Eq. (4.2) is the Hamiltonian of a free massless scalar field and the second is a quadratic interaction Hamiltonian. Owing to the coupling between these terms and those of the homogeneous sector, it is not guaranteed that the hybrid approach is physically feasible beyond the kinematical level, namely, once the constraints are imposed. In the remainder of this section we confirm that one attains in fact a well-defined physical theory.

The explicit form of the action of the Hamiltonian constraint operator on kinematical states is easy to compute as most of the terms in the operator have already been considered in the Bianchi I model or are extensions of well-known operators. The most significant subtleties concern the action of $\widehat{\mathrm{H}_{\text {int }}^{\xi}}$ as this operator includes the sum of all $\hat{a}_{m}^{\dagger} \hat{a}_{-m}^{\dagger}$ terms $(m \neq 0)$, each of which creates an extra pair of "particles" in the modes $m$ and $-m$. However, in spite of the fact that $\widehat{\mathrm{H}_{\text {int }}^{\xi}}$ creates an infinite number of particles, one can prove that it is a well-defined operator in a suitable dense domain of the Fock space [33]. On the other hand, it is straightforward to see that $\hat{\mathcal{C}}_{H}$ leaves invariant Hilbert subspaces which are the tensor product of the superselection sectors of the Bianchi I model times the Fock space. Therefore, as in the Bianchi I model, we can restrict the study to separable Hilbert subspaces whose states have, in the homogeneous sector, quantum numbers $\left(v, \lambda_{\sigma}, \lambda_{\delta}\right)$ with support in discrete sets contained in the positive octant. Let us remember that while $v$ takes values in a semi-lattice of constant step equal to 4 with a minimum equal to $\epsilon \in(0,4]$, the values of $\left(\lambda_{\sigma}, \lambda_{\delta}\right)$ densely cover the positive quadrant of the real plane.

Even though, from a physical perspective, one is only interested in small inhomogeneities which produce a perturbation around the homogeneous Bianchi I background, the hybrid quantum model is well defined and consistent without restrictions on the wave numbers or occupation numbers of the modes, and the evolution can be obtained in much the same manner as in the vacuum Bianchi I model. Using the result that the Bianchi I model in scheme B leads to a well posed initial value problem on the section of constant volume $v=\epsilon$ from which one can evolve the physical state in steps of four units in $v$, one can show that the physical evolution in the hybrid Gowdy model is also (formally) solvable adopting a 
perturbative approach, in which the effect of the interaction term $\widehat{\mathrm{H}_{\text {int }}^{\xi}}$ is treated as small compared to the free Hamiltonian term $\widehat{\mathrm{H}_{\mathrm{o}}^{\xi}}$. Starting with initial data at $v=\epsilon$, one can then find the form of the physical wave functions at $v=\epsilon+4$ in a perturbative expansion. With this data, one can continue the evolution to the next section $v=\epsilon+8$. This procedure can be repeated until one obtains the expression of the physical wave function at the wanted value of $v$ and up to the desired perturbative order. Actually, this perturbative expansion can be understood as an asymptotic expansion in the limit in which the Immirzi parameter tends to infinity. The details of this perturbative expansion are presented in Ref. [33].

The important point here is that the evolution is well defined in this perturbative approach. This is mainly due to the fact that the initial value problem for the vacuum Bianchi I sector is well posed and therefore this result depends upon the proof presented in Sec. III. In this sense, the initial value problems in the vacuum Bianchi I model and the Gowdy $T^{3}$ model are closely related.

\section{DISCUSSION}

In this paper we have first considered vacuum Bianchi I universes with a three-torus topology in the framework of LQC, adopting a new scheme for the improved dynamics which was put forward in Ref. [27]. We have examined some of the aspects of this quantization which had remained unanswered in Ref. [27], like the decoupling of triad components with different orientations under the action of the Hamiltonian constraint, the structure of the superselection sectors in the anisotropies, and the evolution of physical states in terms of the volume as a discrete, internal evolution variable. Then, we have shown that the initial value problem is well posed and completed the quantization of the vacuum Bianchi I model, following scheme B for the implementation of the improved dynamics.

In Sec. IV, we have used the results regarding the vacuum Bianchi I model in order to show that the scheme B hybrid quantization of the linearly polarized Gowdy $T^{3}$ cosmological model is viable. This hybrid quantization provides a first step towards a better understanding of the effect of inhomogeneities in LQC; this is necessary if one wants to eventually obtain predictions about the influence and possible traces of quantum gravity in phenomena like primordial gravitational waves, the cosmic microwave background, and the physics of the early universe in general.

The loop quantization of the Bianchi I model leads to superselection in separable sectors not only for the volume, but also for the anisotropies. Moreover, every superselection sector is restricted to an octant. This is because the Hamiltonian constraint operator, due to appropriate factor ordering choices, does not mix eigenstates of the densitized triad components with different orientations. Moreover, while the superselection sectors in the volume of the Bianchi I universes consist of equidistant points forming a semi-lattice, the superselection sectors in the anisotropies are dense sets in the real semi-axis. On the other hand, the restriction to a definite orientation of the triad components without imposing any kind of boundary conditions, together with the fact that the initial value problem for the evolution is well posed at the minimum value of $v$ (i.e., $v=\epsilon$ ), can be regarded as a realization of a no-boundary prescription for the dynamics. In addition, it is worth emphasizing the result that the discrete evolution in $v$ is well defined starting from the initial section $v=\epsilon$. If this were not the case, the evolution would break down for Bianchi I cosmologies in vacuo for scheme B and, without any reasonable justification, the inclusion of matter would turn out 
to be critical in order for the dynamics of the model to be viable; we have shown that this is not the case.

Concerning the hybrid quantization of the Gowdy model, an important point is that the LQC/Fock split that we have considered assumes that the quantum behavior of the inhomogeneities can be well approximated by conventional quantum field theory methods so that any quantum geometry effects due to the presence of these inhomogeneities can be neglected as perturbatively small. In a true loop quantization of all the gravitational degrees of freedom (i.e., presumably in a reduction of LQG by a suitable incorporation of the symmetries of the Gowdy model), one should treat both the homogeneous and the inhomogeneous sectors in the same manner, that is to say, all of the degrees of freedom should be quantized à la loop. From this perspective, the main assumption in our analysis is that the qualitative results of the hybrid quantization capture the physics of a full loop quantization so long as the inhomogeneities are not directly affected in a significant way by quantum geometry phenomena. It is worth noticing that similar assumptions are implicit in the treatment of other models with matter in LQC inasmuch as matter fields are usually quantized by standard methods rather than by adopting a unified polymer quantization for all of the degrees of freedom, gravitational or not.

Notice nonetheless that, even at this level, one can see that the hybrid quantization approach is sufficient to ensure that the classical cosmological singularities are resolved as the singular states corresponding to vanishing Bianchi I scale factors decouple under the action of the Hamiltonian constraint operator [33, 48]. In addition, the quantum dynamics are well posed as one can use $v$ as an evolution variable and, given the wave function at $v=\epsilon$, one can derive the wave function for all other values of $v$ in the same superselection sector in a perturbative expansion in the interaction term for the inhomogeneities.

There remain many open questions to be addressed, of course, the most important being a numerical study of the evolution of the wave function. This is a very difficult task as even the vacuum Bianchi I model in LQC has not yet been studied numerically in scheme B. We propose to begin with a simpler task and study effective equations associated to the model; this should yield some insight into the most relevant quantum geometry corrections to the classical model [49]. The most important point, however, is to understand inhomogeneities in LQC more deeply and to do this one will have to consider more general inhomogeneous space-times within the framework of LQC in order to obtain physical predictions about our early universe and understand their consequences.

\section{ACKNOWLEDGEMENTS}

The authors are grateful to L.J. Garay, D. Martín de Blas, P. Moniz, J. Olmedo, T. Pawłowski, and specially to J.M. Velhinho for enlightening conversations and discussions. This work was supported by the Spanish MICINN Project FIS2008-06078-C03-03, the Spanish Consolider-Ingenio 2010 Program CPAN (CSD2007-00042), the NSF grant PHY0854743, the George A. and Margaret M. Downsbrough Endowment, the Eberly research funds of Penn State, Le Fonds québécois de la recherche sur la nature et les technologies, and the Edward A. and Rosemary A. Mebus funds. M. M.-B. is supported by CSIC and the European Social Fund under the grant I3P-BPD2006. E. W.-E. thanks the CSIC for their 
hospitality during his visit there.

[1] M. Bojowald, Loop quantum cosmology, Living Rev. Relativity 11, 4 (2008).

[2] A. Ashtekar, An introduction to loop quantum gravity through loop quantum cosmology, Nuovo Cimento B122, 135 (2007).

[3] G.A. Mena Marugán, A brief introduction to loop quantum cosmology, AIP Conf. Proc. 1130, 89 (2009).

[4] A. Ashtekar and J. Lewandowski, Background independent quantum gravity: A status report, Classical Quantum Gravity 21, R53 (2004).

[5] C. Rovelli, Quantum gravity (Cambridge University Press, Cambridge, England, 2004).

[6] T. Thiemann, Introduction to modern canonical quantum general relativity (Cambridge University Press, Cambridge, England, 2007).

[7] M. Bojowald, Absence of singularity in loop quantum cosmology, Phys. Rev. Lett. 86, 5227 (2001).

[8] A. Ashtekar, M. Bojowald, and J. Lewandowski, Mathematical structure of loop quantum cosmology, Adv. Theo. Math. Phys. 7, 233 (2003).

[9] A. Ashtekar, T. Pawłowski, and P. Singh, Quantum nature of the big bang: Improved dynamics, Phys. Rev. D 74, 084003 (2006).

[10] A. Ashtekar, A. Corichi, and P. Singh, Robustness of predictions of loop quantum cosmology, Phys. Rev. D 77, 024046 (2008).

[11] W. Kaminski and J. Lewandowski, The flat FRW model in LQC: the self-adjointness, Classical Quantum Gravity 25, 035001 (2008).

[12] M. Martín-Benito, G.A. Mena Marugán, and J. Olmedo, Further improvements in the understanding of isotropic loop quantum cosmology, Phys. Rev. D 80, 104015 (2009).

[13] V. Taveras, LQC corrections to the Friedmann equations for a universe with a free scalar field, Phys. Rev. D 78, 064072 (2008).

[14] A. Ashtekar and E. Wilson-Ewing, The covariant entropy bound and loop quantum cosmology, Phys. Rev. D 78, 064047 (2008).

[15] A. Ashtekar and D. Sloan, Loop quantum cosmology and slow roll inflation, arXiv: 0912.4093.

[16] E. Bentivegna and T. Pawłowski, Anti-deSitter universe dynamics in LQC, Phys. Rev. D 77, 124025 (2008).

[17] A. Ashtekar, T. Pawłowski, P. Singh, and K. Vandersloot, Loop quantum cosmology of k=1 FRW models, Phys. Rev. D 75, 024035 (2007).

[18] L. Szulc, W. Kaminski, and J. Lewandowski, Closed FRW model in loop quantum cosmology, Classical Quantum Gravity 24, 2621 (2007).

[19] K. Vandersloot, Loop quantum cosmology and the k=-1 RW model, Phys. Rev. D 75, 023523 (2007).

[20] M. Bojowald, Homogeneous loop quantum cosmology, Classical Quantum Gravity 20, 2595 (2003).

[21] M. Bojowald, D. Cartan, and G. Khanna, Lattice refining loop quantum cosmology, anisotropic models and stability, Phys. Rev. D 76, 064018 (2007).

[22] D. Chiou, Loop quantum cosmology in Bianchi type I models: Analytical investigation, Phys. Rev. D 75, 024029 (2007). 
[23] D. Chiou and K. Vandersloot, The behavior of non-linear anisotropies in bouncing Bianchi I models of loop quantum cosmology, Phys. Rev. D 76, 084015 (2007).

[24] D. Chiou, Effective dynamics, big bounces and scaling symmetry in Bianchi I loop quantum cosmology, Phys. Rev. D 76, 124037 (2007).

[25] M. Martín-Benito, G.A. Mena Marugán, and T. Pawłowski, Loop quantization of vacuum Bianchi I cosmology, Phys. Rev. D 78, 064008 (2008).

[26] L. Szulc, Loop quantum cosmology of diagonal Bianchi type I model: Simplifications and scaling problems, Phys. Rev. D 78, 064035 (2008).

[27] A. Ashtekar and E. Wilson-Ewing, Loop quantum cosmology of Bianchi type I models, Phys. Rev. D 79, 083535 (2009).

[28] M. Bojowald, G. Date, and K. Vandersloot, Homogeneous loop quantum cosmology: The role of the spin connection, Classical Quantum Gravity 21, 1253 (2004).

[29] A. Ashtekar and E. Wilson-Ewing, Loop quantum cosmology of Bianchi type II models, Phys. Rev. D 80, 123532 (2009).

[30] M. Bojowald, G. Date, and G. M. Hossain, The Bianchi IX model in loop quantum cosmology, Classical Quantum Gravity 21, 3541 (2004).

[31] E. Wilson-Ewing, Loop quantum cosmology of Bianchi type IX models, Phys. Rev. D 82, 043508 (2010).

[32] A. Corichi and P. Singh, A geometric perspective on singularity resolution and uniqueness in loop quantum cosmology, Phys. Rev. D 80, 044024 (2009).

[33] L.J. Garay, M. Martín-Benito, and G.A. Mena Marugán, Inhomogeneous loop quantum cosmology: Hybrid quantization of the Gowdy model, Phys. Rev. D 82, 044048 (2010).

[34] R.H. Gowdy, Gravitational waves in closed universes, Phys. Rev. Lett. 27, 826 (1971); Vacuum spacetimes with two-parameter spacelike isometry groups and compact invariant hypersurfaces: Topologies and boundary conditions, Ann. Phys. 83, 203 (1974).

[35] V. Moncrief, Infinite-dimensional family of vacuum cosmological models with Taub-NUT (Newman-Unti-Tamburino)-type extensions, Phys. Rev. D 23, 312 (1981).

[36] J. Isenberg and V. Moncrief, Asymptotic behavior of the gravitational field and the nature of singularities in Gowdy spacetimes, Ann. Phys. 199, 84 (1990).

[37] See, e.g., C.W. Misner, A minisuperspace example: The Gowdy T3 cosmology, Phys. Rev. D 8, 3271 (1973); B.K. Berger, Quantum graviton creation in a model universe, Ann. Phys. 83, 458 (1974); Quantum cosmology: Exact solution for the Gowdy T3 model, Phys. Rev. D 11, 2770 (1975); Quantum effects in the Gowdy T3 cosmology, Ann. Phys. 156, 155 (1984); G.A. Mena Marugán, Canonical quantization of the Gowdy model, Phys. Rev. D 56, 908 (1997); M. Pierri, Probing quantum general relativity through exactly soluble midi-superspaces II: Polarized Gowdy models, Int. J. Mod. Phys. D 11, 135 (2002).

[38] A. Corichi, J. Cortez, and G.A. Mena Marugán, Unitary evolution in Gowdy cosmology, Phys. Rev. D 73, 041502 (2006); Quantum Gowdy $T^{3}$ model: A unitary description, Phys. Rev. D 73, 084020 (2006); A. Corichi, J. Cortez, G.A. Mena Marugán, and J.M. Velhinho, Quantum Gowdy $T^{3}$ model: A uniqueness result, Classical Quantum Gravity 23, 6301 (2006); Quantum Gowdy $T^{3}$ model: Schrodinger representation with unitary dynamics, Phys. Rev. D 76, 124031 (2007); J. Cortez, G.A. Mena Marugán, and J.M. Velhinho, Uniqueness of the Fock quantization of the Gowdy $T^{3}$ model, Phys. Rev. D 75, 084027 (2007).

[39] M. Martín-Benito, L.J. Garay, and G.A. Mena Marugán, Hybrid quantum Gowdy cosmology: Combining loop and Fock quantizations, Phys. Rev. D 78, 083516 (2008). 
[40] G.A. Mena Marugán and M. Martín-Benito, Hybrid quantum cosmology: Combining loop and Fock quantizations, Int. J. Mod. Phys. A 24, 2820 (2009).

[41] K. Banerjee and G. Date, Loop Quantization of Polarized Gowdy Model on $T^{3}$ : Classical Theory, Classical Quantum Gravity 25, 105014 (2008).

[42] K. Banerjee and G. Date, Loop Quantization of Polarized Gowdy Model on $T^{3}$ : Kinematical States and Constraint Operators, Classical Quantum Gravity 25, 145004 (2008).

[43] C. Rovelli and F. Vidotto, Stepping out of homogeneity in loop quantum cosmology, Classical Quantum Gravity 25, 225024 (2008).

[44] M. Bojowald, G.M. Hossain, M. Kagan, and S. Shankaranarayanan, Anomaly freedom in perturbative loop quantum gravity, Phys. Rev. D 78, 063547 (2008); Gauge invariant cosmological perturbation equations with corrections from loop quantum gravity, Phys. Rev. D 79, 043505 (2009).

[45] There is a discrepancy in signs between this representation and that of Ref. [27] because, in the latter, $\gamma$ is considered to change sign under internal parity transformations, namely $\gamma=|\gamma| \operatorname{sgn}(v)$, while here, as usual, we treat $\gamma$ as a positive free parameter.

[46] J.M. Velhinho, The quantum configuration space of loop quantum cosmology, Classical Quantum Gravity, 24, 3745 (2007).

[47] J. Cortez and G.A. Mena Marugán, Feasibility of a unitary quantum dynamics in the Gowdy $T^{3}$ cosmological model, Phys. Rev. D 72, 064020 (2005).

[48] In general, other types of singularities do not develop as far as the field, its spatial derivatives, and its momentum remain finite.

[49] G.A. Mena Marugán, T. Pawłowski, and E. Wilson-Ewing, An effective analysis of the hybrid quantization of the Gowdy model (in preparation). 